\title{
Relato de Caso: Otoliquorreia Espontânea em Adulto
}

\section{Case Study Spontaneous Otoliquorrhea in Adult}

\author{
Amanda Costa Rossi*, Juliana Martins de Araujo Cardoso Bertoncello**, Luiz Carlos Scachetti***, \\ Carolina Schäffer***, Aguinaldo Pereira Catanoce ${ }^{* * * *, \text { Silvio Antonio Monteiro Marone }}$ *****.
}

\footnotetext{
* Médica Residente do 3ํㅡㄹ Ano do Serviço de Otorrinolaringologia do Hospital e Maternidade Celso Pierro da Faculdade de Medicina da PUC Campinas.

** Médica Otorrinolaringologista. Médica Especialista Assistente do Serviço de Otorrinolaringologia do Hospital e Maternidade Celso Pierro da Faculdade de Medicina da PUC Campinas.

**** Médico (a) Otorrinolaringologista. Médico (a) Especialista Colaborador (a) do Serviço de Otorrinolaringologia do Hospital e Maternidade Celso Pierro da Faculdade de Medicina da PUC Campinas.

****** Médico Neurocirurgião. Médico Especialista Assistente do Serviço de Neurocirurgia do Hospital e Maternidade Celso Pierro da Faculdade de Medicina da PUC Campinas, do Hospital Vera Cruz e do Hospital Estadual Mário Covas da FMABC.

*******: Professor Titular de Otorrinolaringologia da Faculdade de Medicina da PUC Campinas. Professor Titular de Otorrinolaringologia da Faculdade de Medicina da PUC Campinas e Professor Doutor de Otorrinolaringologia da Faculdade de Medicina da USP.
}

Instituição: Hospital e Maternidade Celso Pierro da Faculdade de Medicina da PUC Campinas.

Campinas / SP - Brasil.

Endereço para correspondência: Amanda Costa Rossi - Rua Com. Luis José Pereira de Queirós, 170 - Apto 141 A - Botafogo - Campinas / SP - Brasil - CEP: 13020-080 - Telefone: (+55 19) 3343-8599 - E-mail: amandarossi@hotmail.com

Artigo recebido em 3 de Maio de 2009. Artigo aprovado em 4 de Agosto de 2009.

\section{RESUMO}

Introdução:

Objetivo:

Relato do Caso:

Comentários Finais:

Palavras-chave:

\section{SUMMARY}

Introduction:

Objective:

Case Report:

Final Comments:

Keywords:
As fístulas liquóricas otológicas espontâneas (FLOEs) são entidades raras. Geralmente manifestam-se em crianças com meningite e perda sensorioneural profunda e em adultos com quadro de otite média com efusão.

Descrever o quadro clínico, o diagnóstico e o tratamento cirúrgico de uma paciente com FLOE. Paciente do sexo feminino, 57 anos, com história de hipoacusia, plenitude aural e tinnitus à direita há 10 anos. Após colocação de tubo de ventilação à direita em outro serviço, iniciou otorreia líquida, transparente e constante em grande quantidade. O diagnóstico foi realizado através da cisternocintilografia, sugestiva de fístula liquórica; e da cisternotomografia que mostrou área de deiscência óssea em região de tegmen tympani à direita, velamento parcial de células mastóideas e de orelha média à direita, além concentração do meio de contraste no espaço sub-aracnoídeo à direita menor em relação ao lado esquerdo. Através de via transmastoídea foi localizada fístula liquórica e meningoencefalocele na região de tegmen tympani à direita. O fechamento da fístula foi realizado através do uso de retalho de músculo temporal, cola de fibrina e Surgicel ${ }^{\circledR}$.

Em adultos com história clínica sugestiva de otite média com efusão recorrente, a hipótese diagnóstica de fístula liquórica deve ser levantada. A investigação deve prosseguir com exames de imagem, destacando-se a cisternotomografia. O tratamento cirúrgico nesta paciente, através da técnica transmastoídea, se revelou eficaz a curto e a longo prazo.

otorreia de líquido cefalorraquidiano, fistula, meningite.

The spontaneous otological CSF leaks are rare entities. Usually they manifest themselves in children with meningitis and deep sensorineural hearing loss and in adults with middle otitis and effusion clinical picture.

To describe the clinical picture, diagnosis and surgical treatment of a patient with CSF otological.

A female sex patient, 57 years old, with hearing loss, aural fullness and right ear tinnitus case history 10 years ago. After insertion of tympanostomy tubes on the right in another service, it began liquid, transparent and constant otorrhea in large quantities. The diagnosis was performed by suggestive cisternoscintigraphy of CSF leak, and by cisternography that showed that the area of bone dehiscence in the tegmen tympani region to the right, partial opacification of mastoid cells and middle ear to the right, besides concentration of contrast medium in sub-arachnoid space to the lower right compared to the left side. Through transmastoid via was located the CSF leak and meningoencephalocele in the region of the right tegmen tympani. The closure of the fistula was accomplished through the use of the temporal muscle flap, fibrin glue and Surgicel ${ }^{\circledR}$.

In adults with symptoms suggestive of recurrent otitis media with effusion, the diagnosis of CSF fistula should be raised. The research should proceed with imaging, highlighting the CTC. Surgical treatment in this patient using the transmastoid technique has proved effective in the short and long term. CSF otorrhea, fistula, meningitis. 


\section{INTRODUÇÃO}

As fístulas liquóricas otológicas são decorrentes principalmente de fatores adquiridos tais como fraturas do osso temporal e de cirurgias envolvendo o osso temporal (1).

As fístulas liquóricas otológicas espontâneas (FLOE) são entidades raras e podem ser agrupadas quanto à idade em dois grupos $(1,2,3)$ :

- Em crianças devido à presença de falhas no osso temporal como deiscências. Nestes casos a manifestação é precoce, entre um e cinco anos de vida, e geralmente cursam com meningite e perda auditiva sensorioneural profunda.

- Em adultos na quinta década de vida, geralmente mulheres obesas, com quadro clínico de otite média secretora.Apresentam uma ou mais áreas de deiscência óssea no tegmen tympani e menos comumente na fossa posterior.

A fisiopatologia da FLOE ainda não está totalmente esclarecida.

Há duas teorias responsáveis, a congênita e a presença de granulações aracnoídeas:

- A teoria de defeito ósseo congênito postula que pequenas comunicações com o tegmen são decorrentes de desenvolvimento embriológico anômalo (4). A passagem do líquor pode ocorrer para orelha interna devido ao aqueduto coclear alargado e assim através de um defeito no modíolo que permite a passagem pelas janelas oval ou redonda (5); ou ainda diretamente para orelha média através de alterações da fissura timpanomeníngea de HyrtL (6)

- A teoria de presença de granulações foi postulada por RICHARD GACEK em 1990 (7) e sugere que granulações aracnoídeas durante o desenvolvimento embriológico terminam em fundo cego na superfície óssea interna da base do crânio. A porção central destas granulações contém líquor e é recoberta frouxamente por uma cápsula fibrosa e por endotélio. Com o tempo, a pressão do espaço subaracnoide causa erosão da cápsula fibrosa e consequentemente óssea das fossas anterior, média ou posterior, resultando em passagem de líquor para orelha média.

O diagnóstico de FLOE depende muito da suspeita clínica. Sugere-se que em qualquer paciente com mais de 50 anos com clínica sugestiva otite média com efusão recorrente deve-se ter como hipótese diagnóstica FLOE entre outras (4).

O objetivo deste artigo e descrever o quadro clínico, o diagnóstico e o tratamento cirúrgico de uma paciente com FLOE em um Serviço de Otorrinolaringologia de um Hospital de atendimento terciário, com residência médica em Otorrinolaringologia.

\section{ReLATO do CAso}

Paciente do sexo feminino, 57 anos, procurou o Serviço de Otorrinolaringologia com história de hipoacusia, plenitude aural e tinnitus à direita há cerca de 10 anos. Negava vertigem. Há cerca de um ano havia sido submetida à colocação de tubo de ventilação à direita em outro Serviço. Desde então iniciou otorreia líquida, transparente e constante em grande quantidade, sem melhora com uso de gotas otológicas. Como antecedentes pessoais referia hipertensão arterial, diabetes mellitus e asma. Negava história de meningite, traumas ou demais cirurgias otológicas.

O exame otorrinolaringológico era normal exceto pela otoscopia direita, em que se evidenciava membrana timpânica opaca com presença de tubo de ventilação por onde drenava abundante quantidade de líquido claro.

A nasofibroscopia não mostrou anormalidades, com óstios tubários livres bilateralmente.

Foi submetida aos seguintes exames complementares.

A dosagem de glicose do líquido da orelha foi 1mg/ dL (proveniente do tubo de ventilação que ainda estava inserido), o que não sugeriu se tratar de líquor.

À audiometria, apresentava perda auditiva condutiva leve à direita.

Quanto aos exames de imagem, a tomografia computadorizada de osso temporal não evidenciou deiscência óssea. A ressonância magnética não trouxe dados relevantes.

Foi então submetida à cisternocintilografia, com sinais sugestivos de fístula liquórica, evidenciando concentração do radiofármaco em região de orelha direita (Figuras 1, 2 e 3). A cisternotomografia, após a introdução de contraste iodado intra-tecal mostrou área de deiscência óssea em região de tegmen à direita, velamento parcial de células mastóideas e de orelha média à direita(Figura 4). Revelou também concentração assimétrica do meio de contraste no espaço sub-aracnoídeo, mostrando-se em menor quantidade no hemisfério cerebral direito (Figuras 5 e 6 ) e corroborando assim com a hipótese de fístula liquórica.

Através de via transmastoídea foi localizada fístula liquórica de cerca de $1 \mathrm{~mm}$ na região do tegmen tympani 


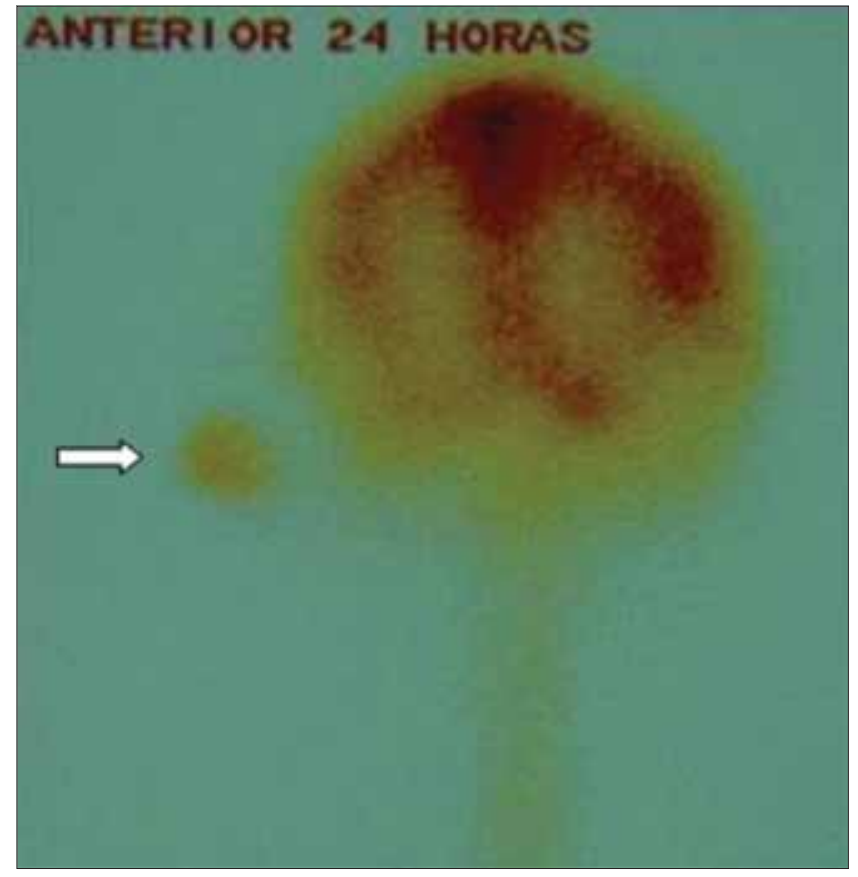

Figura 1. Cisternocintilografia - Anterior - Sugestiva de fístula liquórica em área de orelha direita (seta).

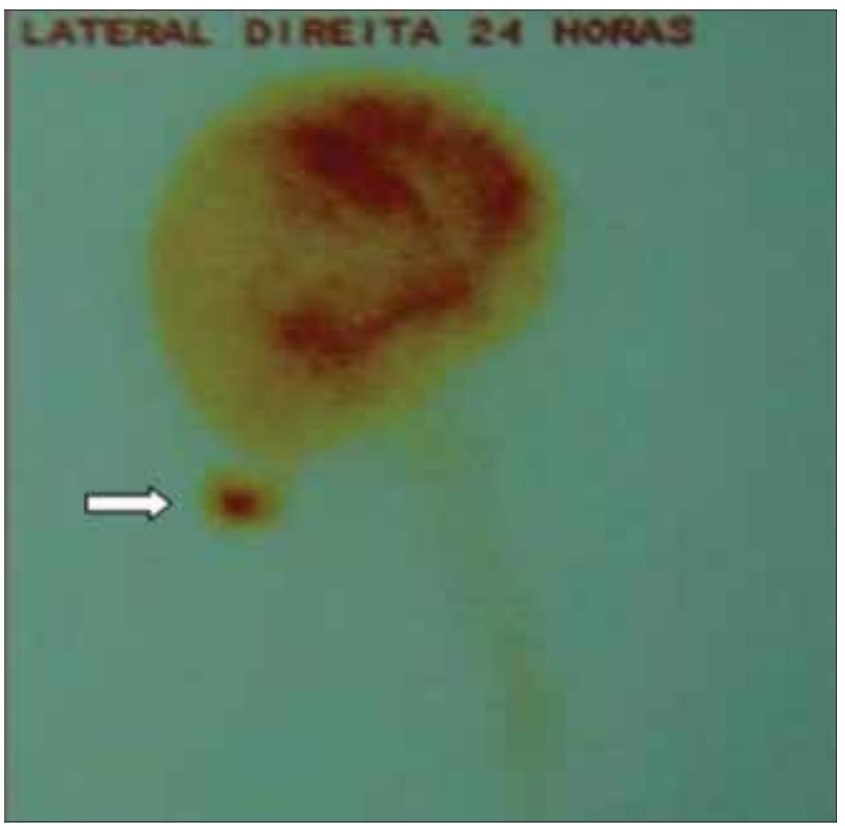

Figura 3. Cisternocintilografia - Lateral Direita - Sugestiva de fístula liquórica em área de orelha direita (setas).

à direita, sendo também evidenciada meningoencefalocele na mesma região. O fechamento da fístula foi realizado através do posicionamento de um retalho de músculo temporal, seguido de colocação de cola de fibrina e Surgicel ${ }^{\circledast}$. Após este procedimento, foi retirado o tubo de ventilação da membrana timpânica direita. Optou-se pela

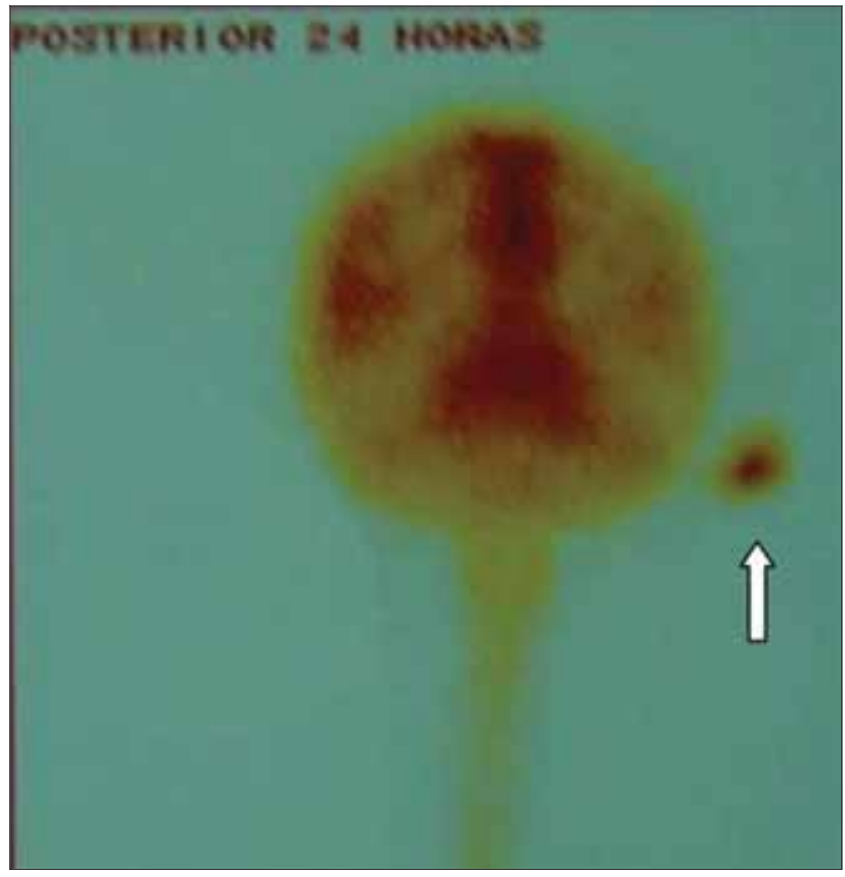

Figura 2. Cisternocintilografia - Posterior - Sugestiva de fístula liquórica em área de orelha direita (seta).

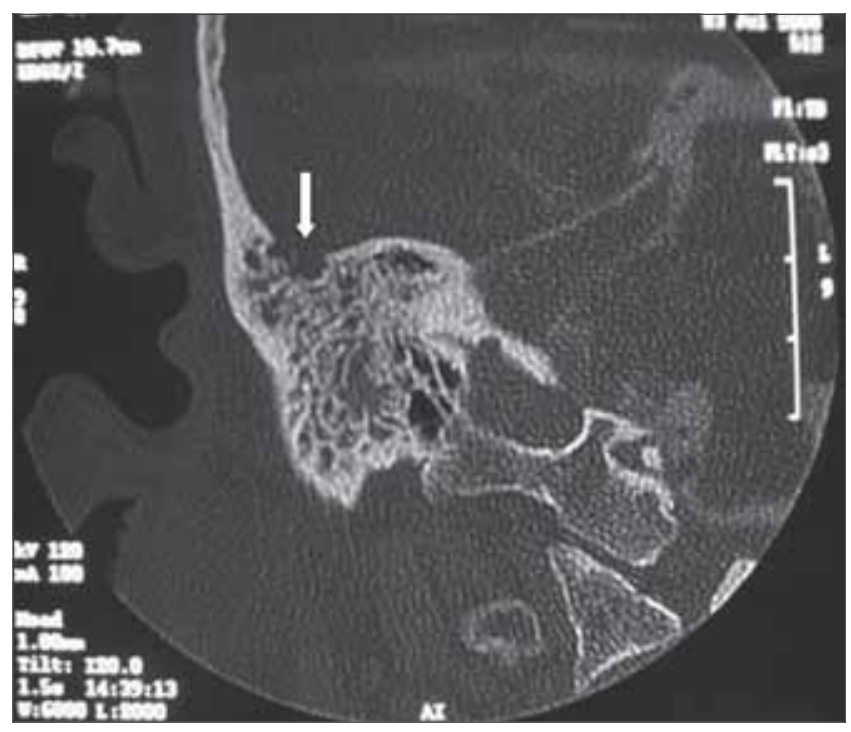

Figura 4. Cisternotomografia de osso temporal (corte coronal) - área de deiscência óssea em tegmen timpani à direita (seta).

retirada do tubo de ventilação pois apesar de a manutenção do mesmo poder evidenciar a permanência de possível otorreia, esta poderia ser verificada pela otoscopia por alterações da membrana timpânica. Além disso, a permanência do tubo de ventilação poderia facilitar a instalação de infecção. 


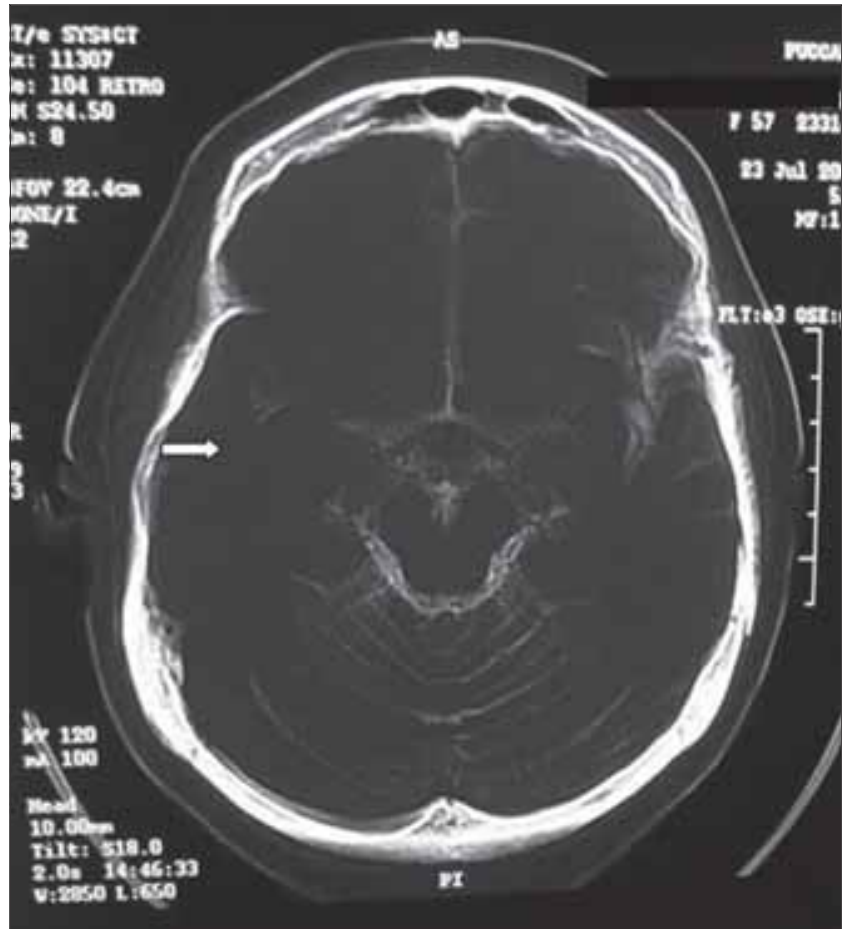

Figura 5. Cisternotomografia de ossos temporais (corte axial) - Concentração do meio de contraste em menor quantidade no espaço sub-aracnoídeo à direita em relação ao lado esquerdo (seta).

A paciente permaneceu 72 horas com drenagem lombar, em decúbito dorsal com cabeceira elevada, em repouso absoluto e em uso de dieta laxativa. Durante internação fez uso de Ceftriaxone 2g IV de 12/12 horas. A paciente recebeu alta no quinto dia pós-operatório. No pósoperatório imediato não mais apresentou otorreia líquida, mantendo-se assim durante o acompanhamento que continua sendo realizado periodicamente, há 12 meses.

Realizou audiometria pós-operatória, que não demonstrou anormalidades.

\section{DisCUSSÃO}

A idade e a forma de apresentação clínica do caso relatado, são condizentes com a teoria de granulação aracnoídea devido à idade de manifestação e ao tipo de quadro clínico apresentado. Quanto ao sexo, a maioria dos estudos $(2,3,8)$ mostra maior incidência em mulheres de meia idade. A suscetibilidade em mulheres não é muito bem entendida, sendo sugerida pressão intracraniana aumentada como possível causa nestas mulheres (2). Há ainda dados na literatura que não relacionam FLOE com o sexo feminino (9).

O diagnóstico precoce da FLOE depende de um alto grau de suspeita clínica $(2,3,4,9)$. Em nosso caso, a suspeita

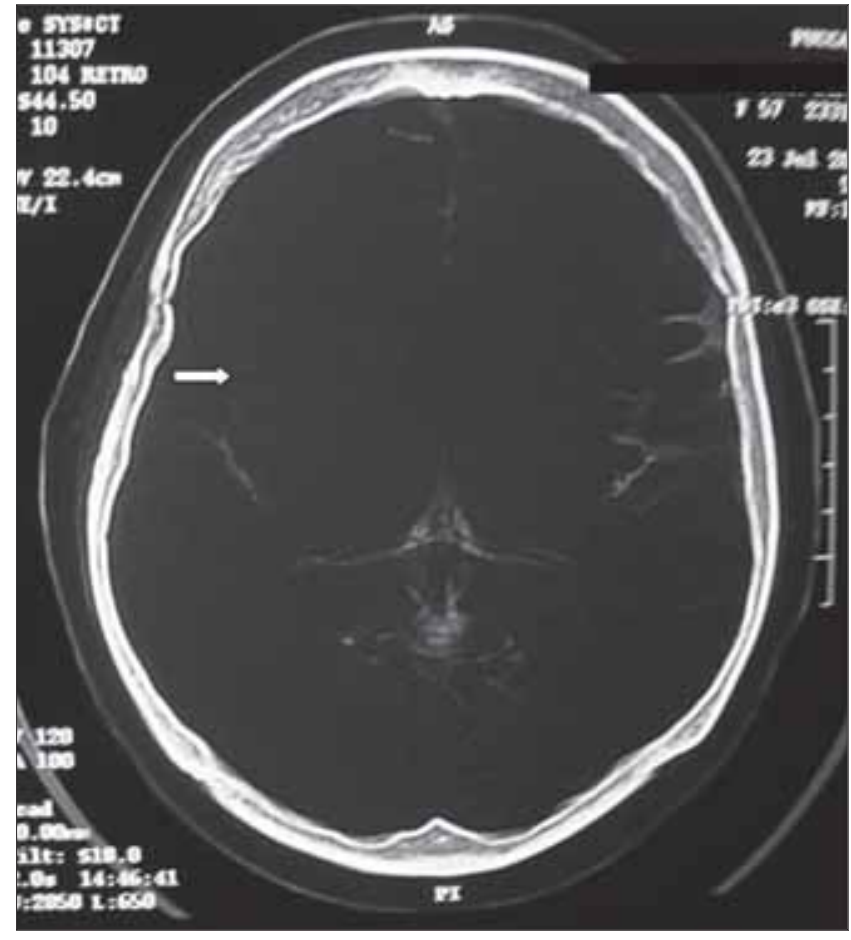

Figura 6. Cisternotomografia de ossos temporais (cortes axial) - Concentração do meio de contraste em menor quantidade no espaço sub-aracnoídeo à direita em relação ao lado esquerdo (seta).

se deu após a realização do exame otorrinolaringológico completo, não havendo justificativa para a ocorrência da referida otorreia.

Podem ser realizados exames laboratoriais, como dosagem de glicose do líquido. Uma concentração de 30 mg/dL de glicose é considerada positiva de líquor, em um paciente com nível normal de glicose. Entretanto, a análise química do líquido obtido da orelha depende da presença de contaminação ou infecção, trazendo uma alta taxa de falsos positivos.Em nosso caso, a dosagem de glicose foi de 0,1 mg/dL, não sendo conclusivo para o diagnóstico. Um teste altamente específico e sensível para fístula liquórica é a dosagem de beta 2 transferrina, uma proteína específica do líquor e que pode ser dosada em quantidades extremamente pequenas de fluido (4), sendo um exame dispendioso. Não dispomos em nosso serviço.

$\mathrm{Na}$ avaliação do paciente com suspeita de fístula liquórica a TC de alta resolução de osso temporal é considerada essencial. A RM não revela deiscências ósseas. A injeção intratecal de radioisótopo foi útil no diagnóstico da presença de fístula, mas não ajudou na localização do local (9).

Quanto à abordagem cirúrgica, vários autores referem à técnica da craniotomia via fossa média para correção de fístula. Há também relatos recentes de 
acesso via transmastoídea e combinação destes acessos $(1,2,3,9,10)$.

O acesso via fossa média permite ampla exposição; assim deiscências grandes (maiores que $2 \mathrm{~cm}$ ), múltiplas ou que se estendem anteriormente ao ápice petroso podem ser corrigidas por esta via (4), poupando a audição. No entanto, é um procedimento com grande potencial para complicações (10). A via transmastoídea possui a vantagem de acessar as fossas média e posterior evitando craniotomia e retração de lobo temporal (4). É também tecnicamente mais fácil de se realizar, sendo um procedimento com menos riscos e complicações, não se mostrando um impedimento para a correção da falha óssea, mesmo de tamanho maior. Assim, pode-se optar inicialmente pela via transmastoídea e na falha deste procedimento utilizar um acesso mais amplo como a craniotomia (10). No caso relatado, optamos pela realização da via transmastoídea como primeira opção por ser um procedimento menos invasivo.

Diversos materiais podem ser utilizados para correção de fístula liquórica: autólogos como fáscia, músculo, cartilagem, gordura e osso; e várias combinações de materiais homólogos e sintéticos $(4,10)$. A técnica de fechamento com múltiplas camadas tem mostrado maiores taxas de fechamento definitivo e menor taxa de recorrência $(2,4)$. No nosso caso utilizamos a técnica de fechamento com várias camadas, com músculo temporal, cola de fibrina e Surgicel ${ }^{\circledast}$.

A derivação lombar pode ser utilizada como adjuvante no pós-operatório por diminuir a pressão intracraniana. Seu uso no pós-operatório é controverso pelo risco de potenciais complicações como meningite, cefaleia ou obstrução do cateter (10). No caso descrito a drenagem lombar foi utilizada por 72 horas, sem complicações.O uso de antibioticoprofilaxia não é referido por muitos autores, porém há relato do uso de Ceftriaxone $1 \mathrm{~g}$ de $8 / 8$ horas (10). No nosso caso optamos por Ceftriaxone 2g de 12/12 horas como medida profilática.

\section{COMENTÁRIOS FINAIS}

Fístula liquórica otológica pode ocorrer na ausência de história de traumas ou meningite. Em adultos com história clínica sugestiva de otite média com efusão recor- rente especialmente sem etiologia para tal, a hipótese diagnóstica de fístula liquórica deve ser levantada. A investigação deve prosseguir com exames de imagem, destacando-se a cisternotomografia. O tratamento cirúrgico nesta paciente, por meio da técnica transmatoídea se revelou eficaz a curto e a longo prazo.

\section{REFERÊNCIAS BIBLIOGRÁFICAS}

1. Wetmore SJ, Herrmann P, Fisch U. Spontaneous Cerebrospinal Fluid Otorrhea. Am J Otol. 1987, 8(2):96102.

2. Rao AK, Merenda DW, Wetmore SJ. Diagnosis and Management of Spontaneous Cerebrospinal Fluid Otorrhea. Otology \& Neurotology. 2005, 26(6):1171-1175.

3. Gacek RR, Gacek MR, Tart R. Adult Spontaneous Cerebrospinal Fluid Otorrhea: Diagnosis and Management. Am J Otol. 1999, 20:770-776.

4. Brown NE, Grundfast KM, Jabre A, Megerian CA, Malley BW, Rosenberg SI. Diagnosis and Management of Spontaneous Cerebrospinal Fluid - Midlle Ear Effusion and Otorrhea. 2004, 114(5):800-805.

5. Montgomery WW. Dural defects of the temporal bone. Am J Otol. 1993, 14:548-551.

6. Schuknecht HF, Gulya AJ. Anatomy of the temporal bone with surgical implications. Philadelphia: Lea \& Febiger, 1986.

7. Gacek RR. Arachnoid Granulation Cerebrospinal Fluid Otorrhea. Annals of Otology, Rhinology and Laryngology. 1990, 99:854-862.

8. Schlosser RJ, Bolger WE. Significance of Empty Sella in Cerebrospinal Fluid Leaks. Otolaryngology Head Neck Surgery. 2003, 128:32-38.

9. May JS, Mikus JL, Matthews BL, Browne JD. Spontaneous Cerebrospinal Fluid Otorrhea from Defects of the Temporal Bone: A Rare Entity? Am J Otol. 1995, 16(6):765-771.

10. Bento RF, Pádua FGM. Abordagem Transmastoídea de Fístula Liquórica em Tegmen Timpânico. Arq Int Otorrinolaringol. 2003, 7(4):250. 